\section{Three Dimensional Imaging of Structure and Flow-Critical to Advances in Microfluidics}

\author{
Carlos Hidrovo ${ }^{1}$, and Terence Lundy ${ }^{2}$
}

${ }^{1}$ Department of Mechanical Engineering, Stanford University

${ }^{2}$ Hyphenated Systems, Burlingame, CA

tlundy@hyphenated-systems.com

Microfluidics, the study of fluid flow through structures with micrometer scale dimensions, is an increasingly important discipline within a number of commercial and industrial applications. One focus of active microfluidic research at the Stanford University Microscale Heat Transfer Laboratories (MHTL) is mass and heat transport in two-phase flows, which has applications in the cooling of integrated circuits and the management of water created in PEM fuel cells. At its core, two-phase microfluidics is the study of interactions between moving liquids and/or gases and/or solids (though not necessarily stationary) structures. Advanced confocal microscopy, with its ability to visualize and measure both flow and structure on a single instrumental platform, will certainly play a key role in the continuing development of microfluidic devices.

\section{Advanced Confocal Microscopy}

Advanced Confocal Microscopy (ACM) refers to the latest generation of high-speed confocal microscopes, such as the Hyphenated Systems 3D MAP currently installed at MHTL (Figure 1). Like conventional optical microscopy, it uses visible light and can provide sub-micrometer aerial resolution. As do all confocal microscopes, it provides sensitivity in the $Z$-direction by using an aperture located in the imaging plane to exclude light rays that originate above or below the object plane. Unlike scanning confocal techniques, which assemble an image from a two-dimensional

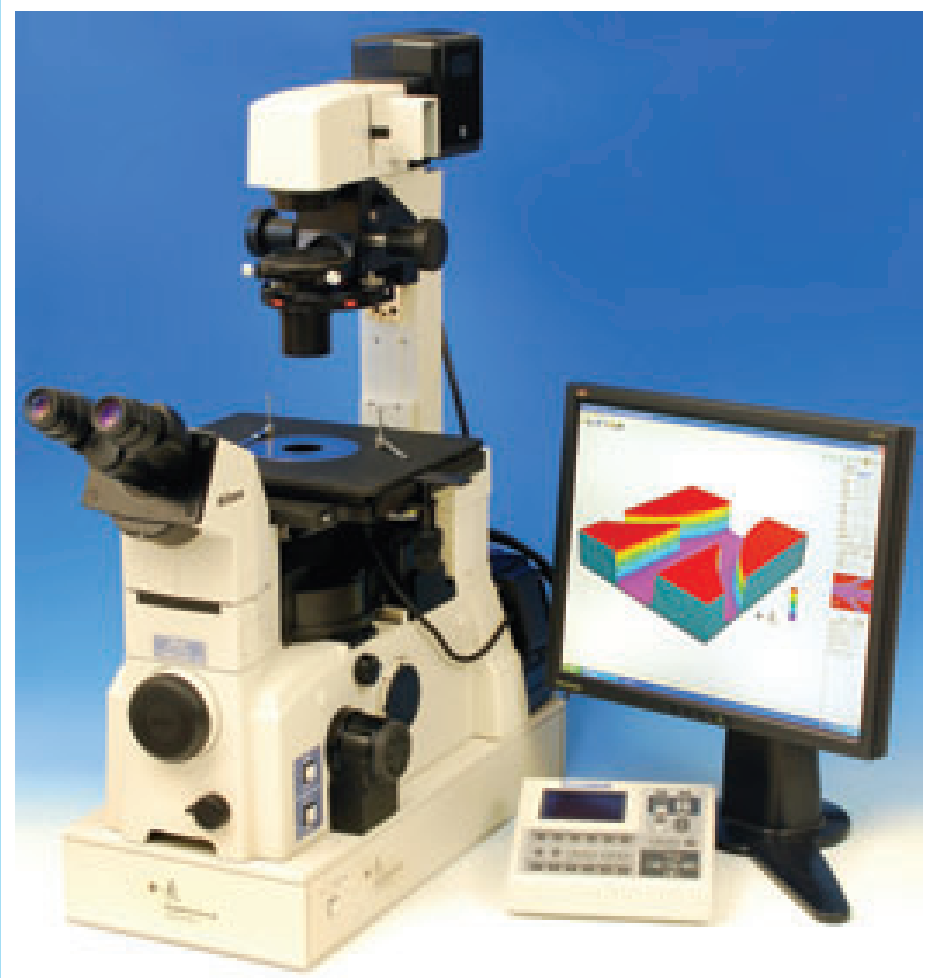

Figure 1: 3D MAP Advanced Confocal Microscope (ACM) installed at Stanford University Microscale Heat Transfer Laboratories (MHTL). array of measurements acquired point-by-point as a finely focused laser scans the object, ACM acquires image information simultaneously through multiple apertures arranged in a spiral pattern on a rapidly spinning disc. Though modern scanning techniques are relatively fast, the multiple apertures and parallel data collection give ACM a fundamental speed advantage in image acquisition.

Although disc-based confocal microscopes have been available for decades, the performance of earlier generations was severely limited by available computing power. With modern processors and programming techniques, ACM can acquire a two-dimensional slice through the sample in a small fraction of a second, and can assemble and render a high-resolution, threedimensional model composed of hundreds of such slices in a few seconds.

Other aspects of the system at MHTL are valuable in microfluidic applications. The ability to selectively exclude signals from overlying transparent layers, such as the glass cover slips often used to enclose microfluidic systems, permits imaging of subsurface features. Open and flexible software architecture permits easy integration with experimental control protocols and ancillary data

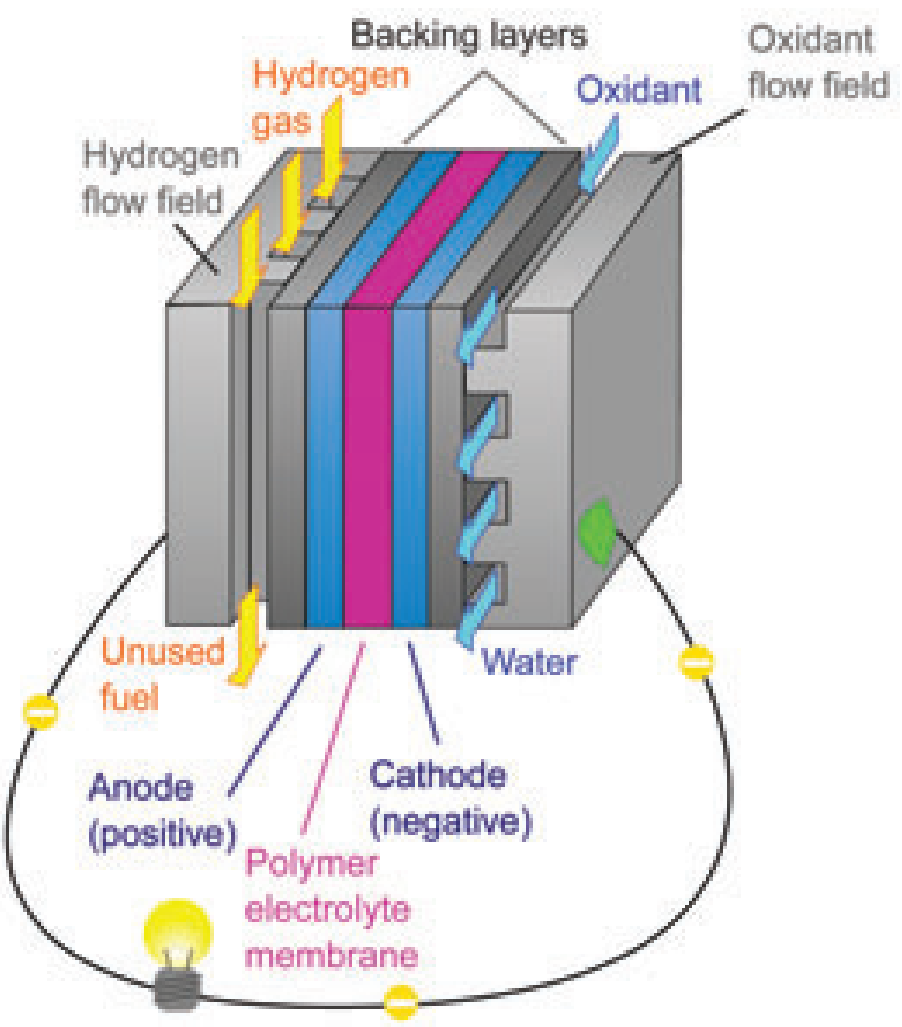

Figure 2: In proton exchange membrane fuel cells the membrane is permeable to protons but not hydrogen or oxygen. Electrons, separated from hydrogen at the anode by a catalyst, travel through an external electrical circuit before recombining with the protons and oxygen at the cathode. Water formed at the cathode must be transported out of the fuel cell.

collection and analysis techniques. An inverted optical system permits observations from below the sample and facilitates access from above for other experimental apparatus.

\section{Flow Characterization}

A number of techniques are available for visualizing and quantifying microfluidic flow using various dyes, bleaches, and marking particles. Caged fluorescence uses a fluorescent dye 


\section{DIATOME diamond knives}

Development, Manufacturing, and Customer Service since 1970

What have we achieved in this period? ultra $45^{\circ}$ the first diamond knife with an absolutely score-free, hydrophilic cutting edge.

semi the first diamond knife for alternating sectioning ultrathin/semithin.

cryo the diamond knife for sectioning at low temperature.

histo the first diamond knife for semithin sections for light microscopy.

ultra $35^{\circ}$ the diamond knife for optimized sectioning results in almost all applications.

STATIC LINE II the ionizer for eliminating electrostatic charging in ultramicrotomy.

cryo-P a cryo knife with a patented platform for section pick up.

cryo immuno the optimized cryo diamond knife for the Tokuyasu technique.

ultra sonic the oscillating diamond knife for room temperature sectioning.

cryotrim 45 and 25 optimizing trimming with diamond blades.

ultra AFM \& cryo AFM the first diamond knives for AFM at room and low temperatures.

cryo $25^{\circ}$ for sectioning frozen hydrated specimens.

What services can we offer you?

I Technical assistance in all fields of ultramicrotomy.

I Free sectioning tests for all types of samples.

I Make use of our many years of experience in perfecting our knives.

I Custom knives, tools, and boats.

I Special purchase programs.

Workshops and training.

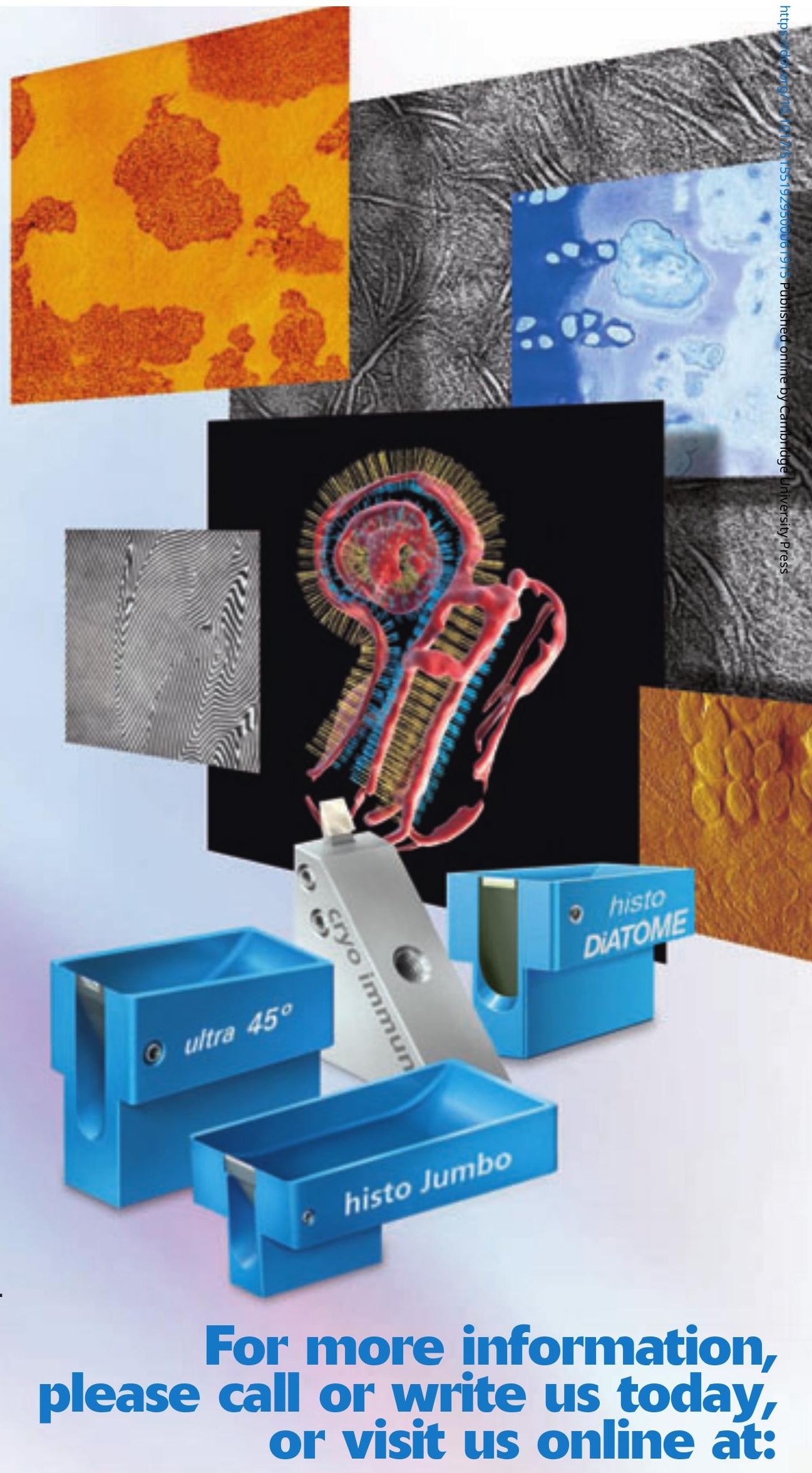

wwwemsdiasum.com

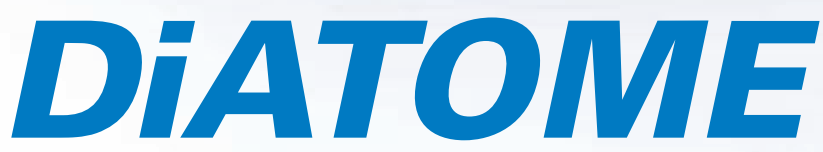

for all your sectioning requirements
P.O. Box $410 \bullet 1560$ Industry Rd. Hatfield, Pa 19440

(215) 412-8390 • Toll Free: 1-(800) 523-5874

Fax: (215) 412-8450 or 8452

email: sgkcck@aol.com• stacie@ems-secure.com 


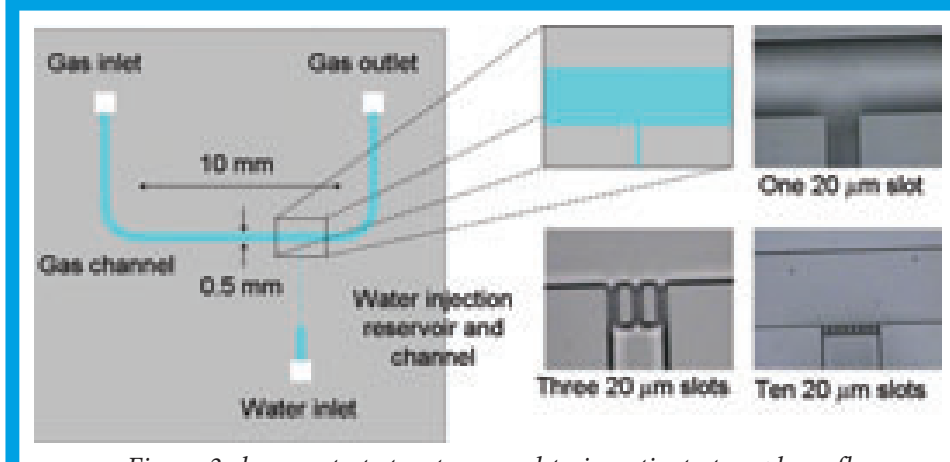

Figure 3 shows a test structure used to investigate two phase flows. Pressurized air flows through the u-shaped channel and fluid is introduced through the small channel perpendicular to it. The liquid port may contain a single or multiple slots to test various liquid injection conditions. The flow structure is mainly determined by the hydrophobicity of the channel walls. As illustrated in figure 4, hydrophilic walls lead to stratified flow conditions while hydrophobic walls tend to induce slug flow. In actual fuel cells channels the gas diffusion layer is usually hydrophobic and the channel walls hydrophilic, creating a mixed flow as shown in cross section at the bottom of the figure.

that is initially in a "caged" non-fluorescent state. After the dye is introduced into the experimental flow, a pulse of ultraviolet light breaks the bonds that cage the fluorescent group. The initial distribution of the uncaged fluorescent marker is thus confined to only the region illuminated by the activating light. At some time after activation, the now fluorescent dye is excited by a brief pulse of laser light, and its distribution captured by an imaging device. Bleached fluorescence is the converse of caged fluorescence. In bleached fluorescence techniques, the fluorescent dye is converted to a non-fluorescent state by a photo bleaching pulse from an argon laser.

Particle image velocimetry (PIV), and closely-related particle tracking velocimetry (PTV), are sophisticated image analysis techniques that characterize flows based on the movement of marker particles. Micro PIV is an adaptation designed for use with microscale flows. PIV cross-correlates sequential images of large numbers of marker particles to derive flow measurements. PTV actually tracks the movements of individual particles between images. PTV has the advantage of being able to separate the effects of random processes (such Brownian motion) and particle interactions from the larger scale flow.

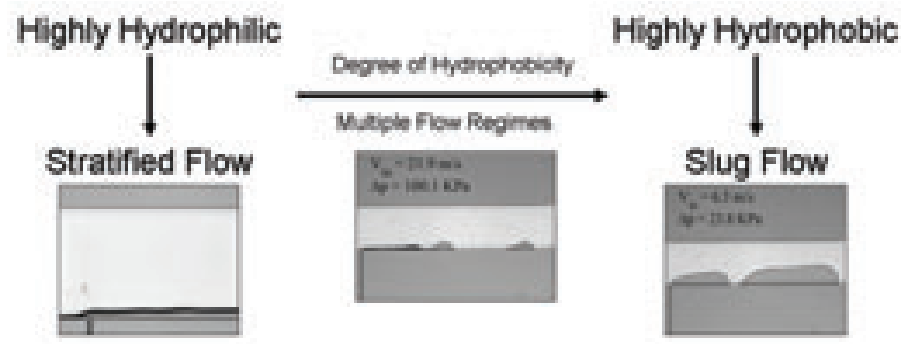

- In actual fuel cells, GDL is usualy hydrophobic and eas channel wal can be hydrophilic or hydrophobic.

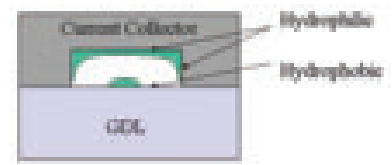

Figure 4 Two phase flows can assume many forms depending on the hydrophobicity of the channel material. In fuel cells the collector is often hydrophilic and the gas diffusion layer (GDL) hydrophobic, resulting in a mixed flow with stratified and slug components.
With ACM it should be possible to develop three-dimensional PIV (and PTV). Conventional particle image velocimetry techniques rely on the use of $2 \mathrm{D}$ images and are thus not capable of resolving the third spatial component of the velocity field. The three-dimensional imaging capability of ACM could enable threedimensional particle image velocimetry, if sequential images can be acquired fast enough to render quasi-instantaneous volume distribution of particles that would allow valid cross correlation and tracking. Three dimensional particle velocimetry is an area of active research and development in a number of laboratories.

\section{Microfluidic Flow}

Fluid flow behavior in micro channels is quite different from that observed at the macroscopic scale in our everyday experience. Single phase microfluidic flows are typically laminar, lacking the turbulence and mixing that occurs readily in larger channels. This is due to the dramatic decrease in characteristic length (and therefore Reynolds number) that reduces the influence of inertial forces relative to viscous forces. Likewise, surface effects, such as surface tension, become predominant as the surface area of the channel walls grows in proportion to channel volume. In addition, when channel dimensions approach the mean free path between fluid molecules collisions and/or characteristic spacing, continuum mechanics and its assumptions break down. One such example is the no-slip boundary condition at the surface of the channel (that flow velocity is zero at the channel wall and increases smoothly with distance from the wall) no longer applies, and slip flow, a regime first explored in high speed aerodynamic analysis, develops. This is particularly true for gases, where the Knudsen number can be much smaller than one in microchannels. Clearly, things are quite different down there and successful development of microfluidic devices for commercial and industrial applications requires a thorough understanding of the physics that govern flow at the microscale.

The applications mentioned above, microelectronic cooling and fuel cell water management, are further complicated by the simultaneous presence of two phases, liquid and gas, in the flow. Two phase flows come in a variety of flavors defined in terms of their characteristics behavior and structure. Stratified flow occurs when the liquid forms a thin continuous layer, usually in contact

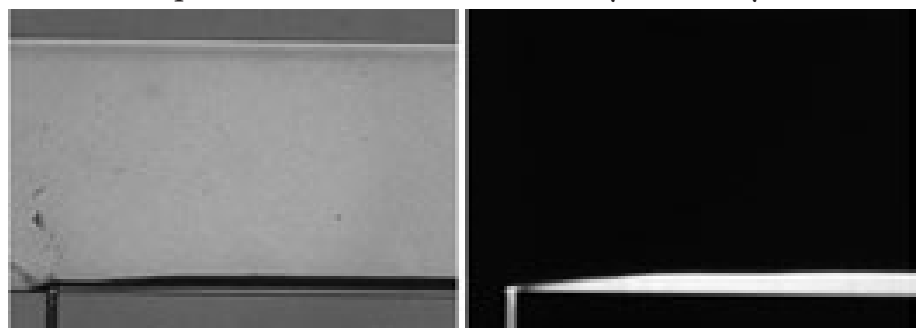

Figure 5: Fluorescent techniques increase contrast in film thickness measurements, but are only two dimensional.

with the channel walls. In slug flow, the liquid occurs in discontinuous droplets along the channel walls. In dispersed flow, small droplets of liquid are dispersed within the gas. Plug flow refers to alternating segments of gas and liquid that fully span the channel. And then there is mixed flow, which can comprise two or more of the other types. And then there is a whole set of descriptors that apply to flows that are predominately liquid with vapor bubbles 


\section{MRS-5}

We are ISO-9000 certified and ISO-17025 accredited Microscopy Calibration Standard

Now you can calibrate from $1,000 \times$ to $1,000,000 \times !$

This is our fourth generation, traceable, magnification reference standard for all types (SEM, FESEM, Optical, STM, AFM, ete.) of microscopy. The MRS-5 has multiple $\mathrm{X}$ and $\mathrm{Y}$ pitch patterns ranging from $80 \mathrm{~nm}( \pm \operatorname{lnm})$ to $2 \mu \mathrm{m}$ and 3 bar targets from $80 \mathrm{~nm}$ to $3 \mu \mathrm{m}$. There is also a STM test pattem.

Free web resource guide!

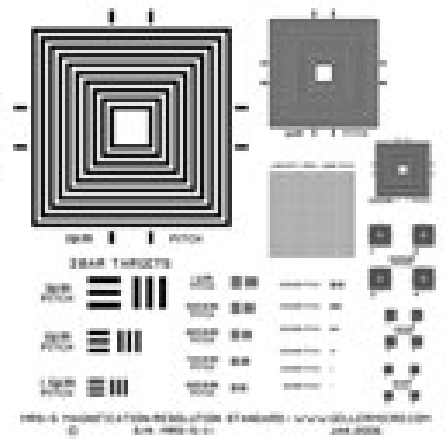

Offering Basic \& Advanced Microscopy Courses

- Polarized Light Microscopy - Asbestos Identification

- Infrared Microspectroscopy - Microscopy of Explosives

- Forensic Hair \& Fiber

- Illicit Drugs \& Excipients

Plus: Customized Courses at your location!

For a complete listing of courses and course descriptions please visit our website at www.mcri.org or call (312) 842-7100

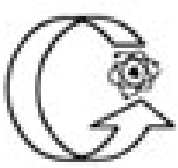

GELLER

MICROẢNALYTICAL

LABORATORY, Inc.

426e Boston St., Topsfield, Ma 01983 www.gellermicro,com

\section{STOP HASSLNG WTH \\ MULTIPLE SERVICE CONTRACTSI}

START by putting all of our instruments under one service contract with MAS (regardless of make or model). Our expert EM SERVICE GROUP has the knowledge and skills to keep your instrument working at its best.

\section{TEM'S / SEM'S}

HITACHI TOPCON

JOEL

ISI

\section{AMRAY CAMBRIDGE}

PREP EQUIPMENT SPECIAL SERVICES

VACUUM COATERS PLASMA ASHERS SPUTTER COATERS ION MILLS
STAGES

BEAM BLANKERS

CUSTOM DEVICES

\section{NEW NEW NEW}

Authorized service representatives

for Gatan preparation equipment and Topcom TEM'S.
Contracts and On-Demand Emergency Service at

Reasonable Rates from

Factory Trained Specialist

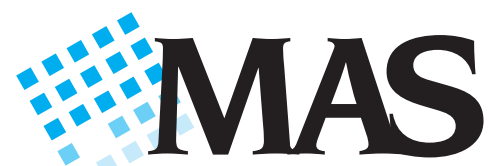

\section{0-421-8451}

3945 Lakefield Court Suwanee, Georgia 30024 770-866-3200 FAX 770-866-3259 ADVANGED ANALYTIGAL PRODUGTS AND SERVIGES 


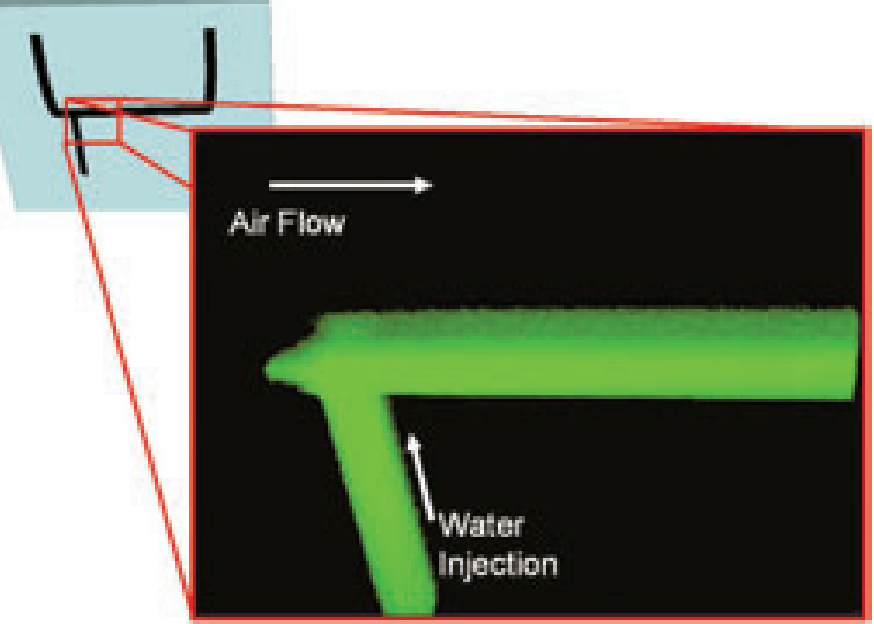

Figure 6 Three dimensional ACM measurements can measure film volume directly.

of various sizes and distributions.

\section{Fuel Cell Applications}

Most fuel cells today are of a type known as polymer electrolyte membrane (or proton exchange membrane) fuel cellsPEMFC (figure 2). As their name implies, they are distinguished by the use of a polymer membrane to mediate the proton exchange that occurs during their operation. The membrane is permeable to protons $\left(\mathrm{H}^{+}\right)$but mostly impermeable to hydrogen and oxygen. PEMFCs are currently the best candidate for transportation and portable applications. They operate at relatively low temperatures $\left(\sim 80^{\circ} \mathrm{C}\right)$, contain no caustic or toxic chemicals, and require only hydrogen and oxygen from air to operate, water being their only by-product.

In a PEMFC the membrane is sandwiched between porous, electrically conductive electrodes, typically a carbon fiber material. The membrane and electrodes are in turn sandwiched between conductive collector plates that contain channels to distribute hydrogen to the anode and to distribute oxygen and extract water and heat from the cathode. The electrochemical reactions that generate current occur on the surface of platinum catalyst particles that coat the carbon fibers of the electrodes at the interface with the membrane. At the anode, hydrogen splits into protons, which traverse the membrane, and electrons, which travel through an electrical circuit to the cathode. At the cathode the protons, electrons and oxygen combine to form water.

Water management is a significant challenge for PEMFC. The membrane requires a certain amount of water to remain permeable to protons. However, water created at the cathode must be removed since its excessive accumulation reduces the ability of gaseous oxygen to reach the catalyst particles, a condition known as cathode flooding. Capillary force is the predominant driving mechanism that moves water through the porous cathode and into the distribution channels of the collector plate. The focus of our research at the MHTL is the transport of liquid water, in a two phase flow propelled by the air supply, through these micro scale channels.

Figure 3: A silicon based microfabricated experimental structure was developed to investigate the pressure-driven, two-phase
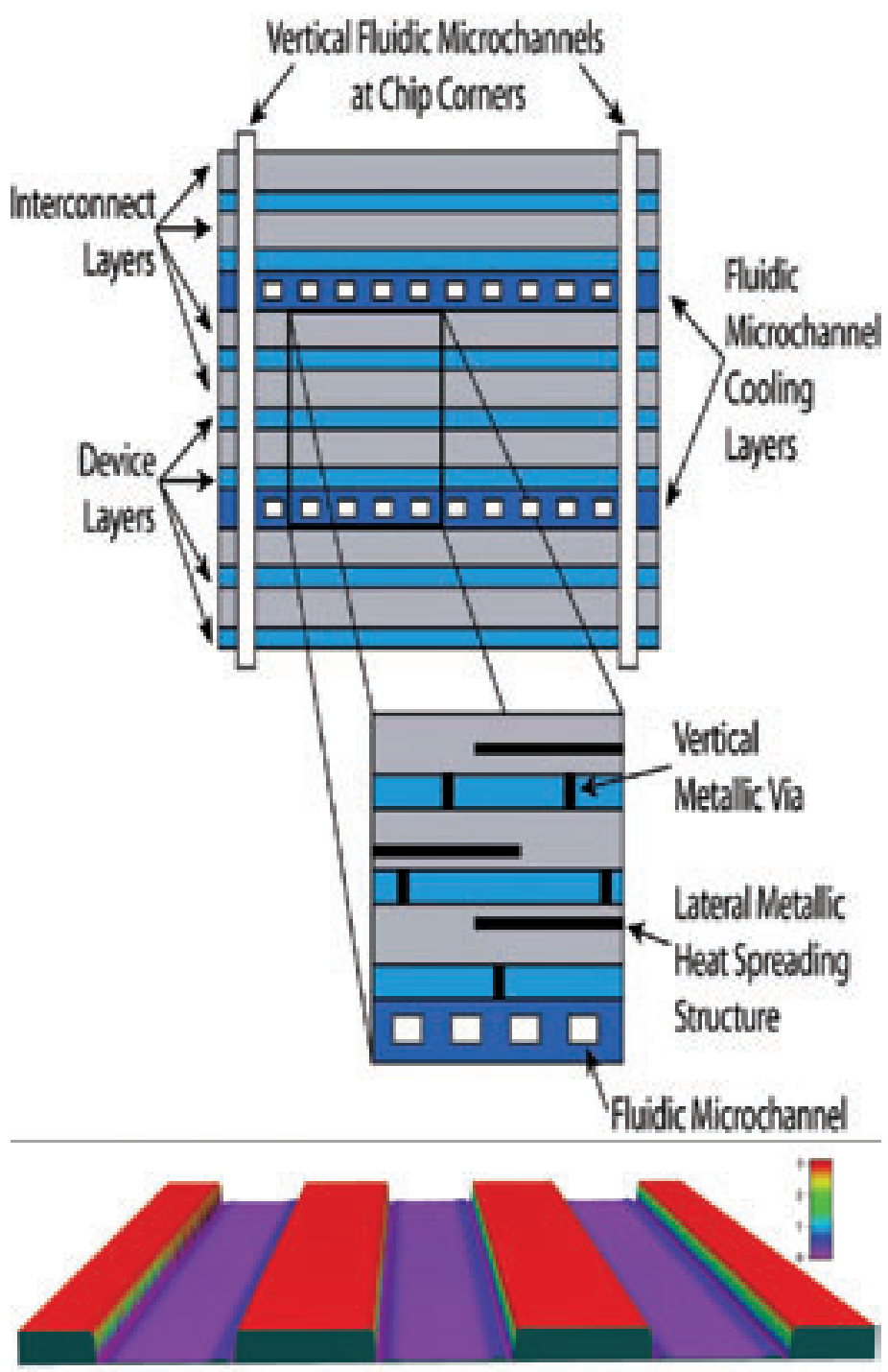

Figure 7 Top drawing -- Heat dissipation is a major challenge in the development of three dimensional integrated circuits (3DIC) that stack multiple dies. Microfluidic channels integrated into the interconnect architecture to circulate cooling fluids are a potential solution. Allowing the fluids to boil would increase the heat transport efficiency but requires a thorough understanding of two phase flows in microchannels. Bottom image

- 3D MAP image of fluidic microchannels, each channel approximately

12 microns in width.

flow that removes water from the fuel cell (structures fabricated by Fu-Min Wang).

Fluorescence microscopy is a central tool for flow characterization. The sample fluid can be "stained" with a fluorescent dye or seeded with fluorescent, micrometer size (or smaller) particles, that emit light of a known wavelength when illuminated by light of another specified wavelength. Filters eliminate the illuminating and background light, making the fluorescence easy to distinguish within the image. Fig 5 compares a conventional white light image with a fluorescence image of a stratified flow. The enhanced image contrast provided by the fluorescence can be used for highly accurate quantitative film thickness measurements.

Although fluorescence improves the quality of such measurements, it is only two dimensional-it provides film thickness in the image plane only. Characteristics such as liquid volume would be better measured with a three dimensional techniques such a 

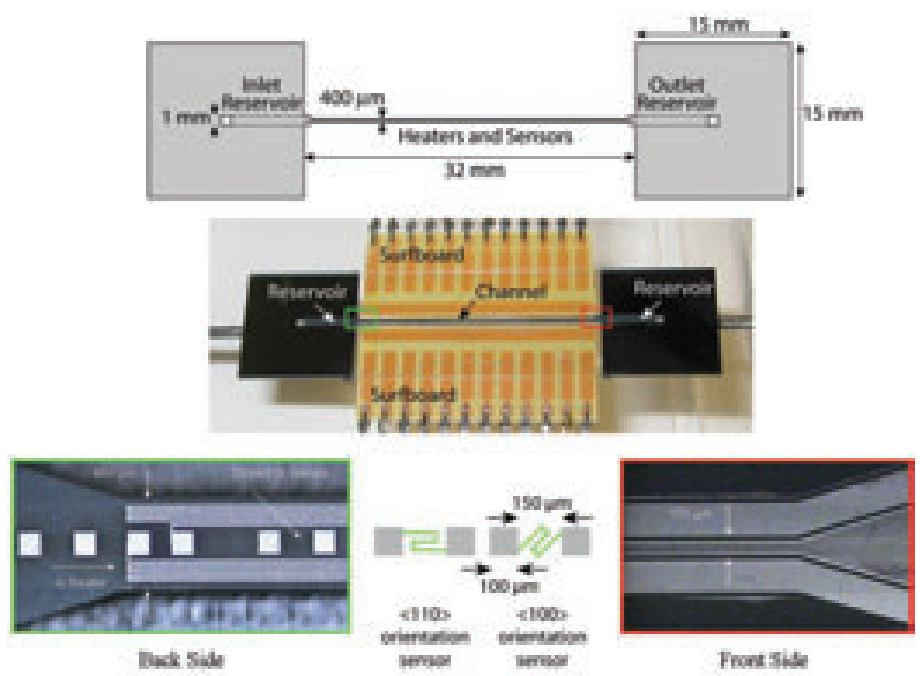

Figure 8 An isolated microchannel with integrated heating and temperature sensors is used to explore the behavior of boiling liquids in microchannels. (Images courtesy of Roger Flynn)

confocal microscopy (Fig 6).

Capillary forces play a predominant role in determining the behavior of two phase flows. Contact angle measurements that quantify surface tension can be difficult to acquire within a micro channel. The quantitative, three-dimensional imaging capabilities of ACM could permit fast and accurate measurements of contact angles, even with the limited accessibility of many microfluidic structures.

\section{Microelectronic Cooling}

As microelectronic devices have been scaled to smaller dimensions and higher speeds, power dissipation has become a major
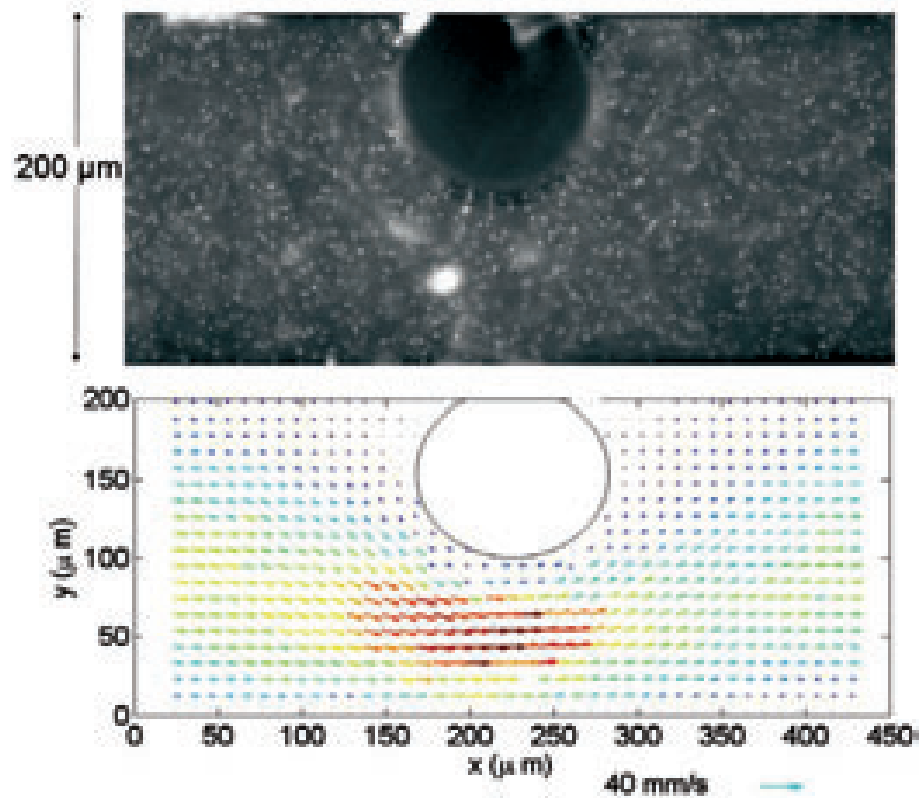

Figure 9 shows $\mu$ PIV imaging results obtained from flow visualization around a growing bubble. The tracking particles used for micro PIV are clearly visible in the image at the top. The arrows in the lower plot indicate the speed and direction of flow across the two-dimensional section defined by the focal plane of the imaging system. Since the imaging system is not sensitive to movement in the $Z$-direction, some of the errors in the $2 \mathrm{D}$ field are associated with the projection of the true, three-dimensional velocity vectors onto the plane of the image. In theory, ACM could support threedimensional micro PIV measurements, if images can be acquired with sufficient speed. challenge. This problem is now being compounded by the move to extend the conventional planar architecture that has dominated integrated circuit design for decades into the third dimension by stacking completed circuits on top of the other. Although the approach has obvious benefit in increasing the density of computing power, it also multiplies the electrical power that must be removed.

Currently, the most common approach to three-dimensional interconnect architecture is simply stacking the chips within a package and connecting them with wire bonds. A number of other techniques are in development that will connect multiple dies with through-silicon-vias (TSV) that pass through the chip itself. Some experts have speculated that we may eventually be able to connect as many as a hundred circuits in three-dimensional integrated circuits (3DIC), providing enormous increases in computing power. Clearly, a successful heat dissipation strategy is a prerequisite.

One solution currently under consideration is the incorporation of microchannels in the interconnect architecture to conduct cooling fluid through the circuit. The cooling efficiency of such an approach can be enhanced if the fluid boils in the process, thereby taking advantage of the latent heat of the phase transition to increase the total removal. However, the formation of bubbles in the boiling fluid also has the potential to interfere with cooling, if the bubbles, which nucleate on the channel walls, grow large enough to block the flow before they detach and become entrained in the flowing liquid.

Ongoing research at the MHTL focuses on the micro scale physics of bubble nucleation, growth and detachment. If researchers can understand the forces exerted on a bubble by the fluid that lead to the bubble's detachment, it may be possible to determine conditions that will prevent the formation of large bubbles and ensure reliable cooling.

\section{Conclusion}

Though microfluidic devices are already commonplace in applications ranging from ink jet printers to labs on chips, much work still remains to gain a detailed understanding of fluid behavior on the microscale. New applications that could have far reaching effects include fluid transport in fuel cells and cooling in microelectronic devices. Both of these involve these use of two-phase flows in which three dimensional phenomena play an important role. Advanced confocal microscopy can provide fast, three-dimensional visualization of both structure and flow at the micro scale and promises to make significant contributions to the continuing development of microfluidic technologies.

\section{Acknowledgements:}

All of the research mentioned in this article was performed in Professor Kenneth Goodson's Microscale Heat Transfer Laboratory at Stanford University. Special acknowledgement to Prof. Evelyn Wang of MIT, whose Ph.D. work at Stanford University in microchannel boiling bubble dynamics is portrayed in this article.

Carlos Hidrovo is now Assistant Professor, Mechanical Engineering Department, University of Texas at Austin. Hidrovo@ mail.utexas.edu. 